\title{
Ketamine as an adjunct to patient-controlled analgesia: why, for whom, and how much?
}

\author{
Anuj Bhatia, MBBS, MD
}

Received: 29 August 2015/Revised: 19 October 2015/ Accepted: 19 November 2015/Published online: 25 November 2015

(C) Canadian Anesthesiologists' Society 2015

Ketamine is a phenylpiperidine derivative that has been available for over six decades. For many years, it has been used to provide analgesia and anesthesia in battlefield and emergency room settings, not to mention in veterinary medicine. Though its role as an anesthetic agent in the operating room is well known, its use has arguably been on the decline, in part because of its psychotropic and sympathomimetic adverse effects. Nevertheless, a resurgence in its use is now being seen, in some measure due to its multimodal mechanisms of analgesia: antagonism of N-methyl-D-aspartate (NMDA) excitatory glutamate receptors that play a prominent role in facilitating nociceptive transmission; enhancement of descending inhibition, and anti-inflammatory effects in the central nervous system. ${ }^{1-3}$ As a result, ketamine has now been increasingly used for a range of effects, including antinociceptive effects for treating pain in its various forms (acute, chronic, neuropathic, cancer-related) as well as for addressing treatment-resistant depression. ${ }^{4,5}$

Recent investigations have focused on the role of subanesthetic doses of ketamine in improving analgesia and the potential for reducing requirements for other analgesics, especially opioids. In this issue of the Journal, Wang et al. present results of their systematic review and meta-analysis of 36 randomized-controlled trials (RCTs) in

\footnotetext{
A. Bhatia, MBBS, MD $(\bowtie)$

Department of Anesthesia and Pain Management and Institute of

Health Policy Management and Evaluation, University of

Toronto, Toronto, ON, Canada

e-mail: anuj.bhatia@uhn.ca
}

\section{A. Bhatia, MBBS, MD}

University Health Network-Toronto Western Hospital, Mount Sinai Hospital, and Women's College Hospital, Toronto, ON, Canada
English and Chinese languages. The included studies, enrolling over 2,500 patients, investigated the effects of combining intravenous ketamine with opioid (morphine or hydromorphone) intravenous patient-controlled analgesia (PCA). ${ }^{6}$ Though others have reviewed this topic in the past ten years using either qualitative or quantitative approaches, ${ }^{7-12}$ the meta-analysis by Wang et al. represents the most comprehensive effort to date for evaluating the effects of adding ketamine to opioid PCA. Inclusion criteria were restricted only to those RCTs using subanesthetic doses of ketamine, defined as an intramuscular dose of $\leq 2$ $\mathrm{mg} \cdot \mathrm{kg}^{-1}$, or intravenous/epidural doses of $\leq 1 \mathrm{mg} \cdot \mathrm{kg}^{-1}$, or an intravenous infusion rate of $\leq 20 \mu \mathrm{g} \cdot \mathrm{kg}^{-1} \cdot \mathrm{min}^{-1}$ (or $1.2 \mathrm{mg} \cdot \mathrm{kg}^{-1} \cdot \mathrm{hr}^{-1}$ ). The meta-analysis included RCTs evaluating ketamine administered peri- and postoperatively, either mixed with the opioid in the PCA pump or administered as a stand-alone infusion. Only three of the RCTs evaluated the impact of adding ketamine to hydromorphone PCA. There was a wide range of surgical settings, including orthopedic, thoracic, cardiac, and abdominal operations. Overall, the meta-analysis included good-quality RCTs with moderate-high confidence in the estimates.

The addition of ketamine was associated with small (i.e., $4-13 \%)$ decreases in pain scores as well as reductions in morphine consumption ( 5 and $20 \mathrm{mg}$ at the end of the first and third postoperative days, respectively). Nevertheless, a dose-response analgesic effect for ketamine could not be ascertained. There were no increases in the anticipated adverse effects of ketamine (e.g., hallucinations, dysphoria, diplopia, and sympathetic stimulation). Furthermore, the addition of ketamine reduced the incidence of postoperative nausea and vomiting (PONV), though with the caveat that there was a publication bias for this observation. This suggests that the number of published 
studies reporting a reduction of PONV with the use of ketamine was higher than expected compared with studies that showed no effect or an increase in the incidence of PONV with ketamine.

Previous systematic reviews have reported similar findings regarding reductions in the requirement of postoperative opioids with the use of ketamine., ${ }^{9,10,12}$ These prior reviews echo Wang et al.'s conclusions that subanesthetic doses of ketamine have minimal adverse effects.

Several questions remain unanswered or are newly generated from this review. First, what "subanesthetic" dose of ketamine should be used to optimize analgesic benefits and minimize adverse effects? The term "subanesthetic" simply implies a dose that is not sufficient to induce anesthesia with all its usual components (i.e., amnesia, analgesia, unconsciousness, and immobility), but a consensus regarding the precise dosage has not been determined. A commonly reported dosing regimen includes a pre-incision slow bolus of intravenous ketamine 0.25-0.5 $\mathrm{mg} \cdot \mathrm{kg}^{-1}$ followed by an infusion of $0.25-0.5 \mathrm{mg} \cdot \mathrm{kg}^{-1} \cdot \mathrm{hr}^{-1}$ that is discontinued an hour or so before the end of surgery. ${ }^{13}$ It is important to recognize that this dose is significantly lower than the upper limit $\left(1.2 \mathrm{mg} \cdot \mathrm{kg}^{-1} \cdot \mathrm{hr}^{-1}\right)$ used by Wang et al. in their meta-analysis to describe subanesthetic doses of ketamine. Sedation or other psychotropic effects in the postoperative period are unusual with doses in this range. Nevertheless, there is a lack of RCTs evaluating the most appropriate dose of ketamine infusion for efficacy and safety in the perioperative context.

Second, why is it that RCTs and reviews have not established a dose-response relationship for the analgesic benefit from adding ketamine to PCA, especially in opioidnaïve patients? Is this due to methodological limitations (i.e., RCTs with a small sample size; heterogeneity in systematic reviews and meta-analyses) or the possibly limited role of NMDA receptors in acute postoperative pain? The role of NMDA receptors in the development of central sensitization would certainly suggest proliferation/ upregulation of these receptors in proportion to the duration of pain ${ }^{14}$ and the utility of ketamine in chronic pain states such as neuropathic, chronic, cancer-related, and persistent postoperative pain. This may explain our inability to exploit the analgesic potential of ketamine in the healthy patient in the relatively short perioperative period.

Third, did the RCTs included in Wang et al.'s metaanalysis underreport the adverse effects? Most of the RCTs in their review were of modest size; study durations were limited to the early postoperative period, and more importantly, most RCTs did not rigorously monitor for adverse effects of ketamine. That being said, this metaanalysis showed a reduction in the incidence of PONV in patients who received ketamine, a finding replicated in other reviews. ${ }^{4,11}$ The most likely explanation is a ketamine-associated decrease in the use of opioids. This observation draws our attention to the important issue of evaluating the impact of analgesic adjuncts on alleviating potential adverse effects of opioids (e.g., respiratory depression, hyperalgesia, tolerance, dependence, and role in the development of persistent postoperative pain). Reductions in the intensity and frequency of these adverse effects should be evaluated as primary outcomes in adequately powered clinical trials.

Fourth, what are the possible long-term positive (e.g., reduction in persistent postoperative pain) and negative (e.g., cognitive impairment with long-term use/abuse) implications of using ketamine in the perioperative period? ${ }^{2,15}$ While ketamine has been suggested as an important weapon in the armamentarium for preventive analgesia, ${ }^{7}$ does this benefit outweigh the risks?

So quo vadis? Perhaps future directions should consider the use of ketamine for specific "at-risk" populations in the postoperative period. Examples include patients on high doses of opioids who are likely to display features of opioid-induced hyperalgesia or tolerance. ${ }^{16} \mathrm{~N}$-methylD-aspartate receptors are implicated in the pathogenesis of these phenomena. Other groups of individuals who could benefit from administration of ketamine are those who have features of neuropathic pain ${ }^{17}$ (e.g., patients undergoing spine surgery for axial and/or radicular pain $)^{18,19}$ or those who have a history of significant adverse effects (e.g., PONV) from opioids. We should also consider using ketamine for individuals who are likely to develop persistent postoperative pain (PPP) of neuropathic etiology. Examples of populations at risk for this syndrome include those undergoing mastectomy, amputation, and thoracotomy. Ketamine may be useful as an adjuvant in this setting because it can reduce acute postoperative pain (an important risk factor for PPP) through its antagonism at the NMDA receptor. ${ }^{20}$

Antagonism of NMDA receptors is also responsible for the antidepressant effects of ketamine, ${ }^{5}$ opening an interesting avenue for research among patients with depression (subclinical or overt). Will ketamine treatment result in an improvement in depressive symptoms as well as in lower pain scores? Would pain scores be lower because of the known euphoric effects of ketamine? Evaluating mood and depression in patients undergoing surgery through the use of validated tools and advanced functional brain imaging techniques, both prior to and after the use of ketamine, may yield useful insights into the link between analgesia and psychological status.

It would be remiss not to mention that the minor benefit obtained from adding ketamine should be weighed against the potential for adverse effects (psychotropic and sympathomimetic effects) in the postoperative period. There is also evidence of hepatic and urinary bladder 
dysfunction in individuals who have either abused ketamine or received multiple infusions. ${ }^{2}$

Several other adjuncts (e.g., magnesium, lidocaine, gabapentinoids, and alpha- 2 agonists) ${ }^{21}$ to opioid analgesia have recently been explored in the perioperative setting, with mixed results from RCTs and observational research evaluating these adjuncts. ${ }^{22}$ Clinicians wanting to employ the variety of opioid adjuncts in the treatment of acute postoperative pain should pose the following questions. Is use of the adjunct associated with a clinically relevant impact on the quantity and quality of pain? Does the addition of the adjunct lead to a reduction in requirements for other analgesics, especially those associated with significant adverse effects in susceptible populations (e.g., pediatric, elderly, or hemodynamically unstable patients or those with renal or hepatic dysfunction)? Does the adjunct have a favourable adverse-effect profile? In other words, do the common adverse effects of the medication have a minimal impact and are serious adverse effects rare? Is the administration of the adjunct feasible? Can the adjunct be given as a one-time bolus or as an infusion, or are both methods required? Are there any possible pharmacokinetic or pharmacodynamic interactions with other medications (e.g., acetaminophen, non-steroidal anti-inflammatory drugs, opioids, antibiotics)? Does the adjunct have potential for preventive analgesia? ${ }^{7}$ Are attendant "co-domains" of pain affected favourably by the adjunct? Anxiety, depression, and catastrophizing have been shown to prognosticate, exacerbate, and perpetuate severe postoperative pain. ${ }^{23}$ Use of validated tools to measure these traits at pre- and postintervention stages would help in understanding the mechanistic foundations of a medication's analgesic effect.

Though ketamine and other adjuvants have the potential to improve perioperative analgesia, we continue to search for an ideal effective adjuvant that yields maximum analgesic benefit with minimal adverse effects. We also need a balance between procedure- and patient-specific considerations while planning perioperative analgesic regimens. This requires well-designed and adequately powered clinical trials that involve use of validated tools to measure all the important aspects of perioperative pain.

\section{La kétamine en ajout à une analgésie contrôlée par le patient : pourquoi, pour qui et combien?}

La kétamine est un dérivé de phénylpipéridine disponible sur le marché depuis plus de six décennies. On l'a utilisé pendant de nombreuses années pour procurer une analgésie sur les champs de bataille et dans les services d'urgence, sans oublier son utilisation en médecine vétérinaire. Alors que nous connaissons bien son rôle comme agent anesthésique en salle d'opération, son utilisation semble avoir diminué notamment à cause de ses effets secondaires psychotropes et sympathomimétiques. Néanmoins, une retour de son utilisation est maintenant constatée dans une certaine mesure en raison de son mécanisme analgésique multimodal: antagonisme des récepteurs du glutamate activés par le $\mathrm{N}$-méthyl-D-aspartate (NMDA) qui jouent un rôle majeur dans la facilitation de la transmission nociceptive; renforce ment de l'inhibition descendante et effets anti-inflamma toires sur le système nerveux central. ${ }^{1-3}$ En conséquence, la kétamine est maintenant de plus en plus utilisée pour une série d'effets incluant ses effets antinociceptifs pour le traitement de la douleur dans ses différentes formes (aiguë, chronique, neuropathique, cancéreuse) ainsi que pour son action contre la dépression résistante au traitement. ${ }^{4,5}$

Des recherches récentes se sont concentrées sur le rôle de doses infra-anesthésiques de kétamine dans l'amélioration de l'analgésie et sur la possibilité de diminuer les besoins d'autres analgésiques, plus particulièrement des morphiniques. Dans ce numéro du Journal, Wang et coll. présentent les résultats de leur étude et méta-analyse systématique de 36 essais cliniques randomisés (ECR) en langues anglaise et chinoise. Les essais retenus, incluant plus de 2500 patients, ont étudié les effets de l'association de la kétamine intraveineuse avec une analgésie intraveineuse contrôlée par le patient (ACP) utilisant des opioïdes (morphine ou hydromorphone). ${ }^{6}$ Bien que d'autres se soient intéressés à ce sujet au cours des dix dernières années en utilisant des approches qualitatives ou quantitatives, ${ }^{7-12}$ la méta-analyse de Wang et coll. représente le travail le plus complet à ce jour pour évaluer les effets de l'addition de la kétamine à l'ACP par opioïdes. Les critères d'inclusion étaient limités aux seuls ECR utilisant des doses infra-anesthésiques de kétamine, c'est-à-dire une dose intramusculaire $\leq$ $2 \mathrm{mg} \cdot \mathrm{kg}^{-1}$ ou une dose intraveineuse/péridurale $\leq 1 \mathrm{mg} \cdot \mathrm{kg}^{-1}$, ou un débit de perfusion intraveineuse $\leq 20 \mu \mathrm{g} \cdot \mathrm{kg}^{-1} \cdot \mathrm{min}^{-1}$ (ou $1,2 \mathrm{mg} \cdot \mathrm{kg}^{-1} \cdot \mathrm{h}^{-1}$ ). La méta-analyse a inclus des ECR évaluant l'administration de kétamine en péri- et postopératoire, mélangée aux opioïdes dans la pompe d'ACP ou dans une perfusion indépendante. Seuls trois des essais cliniques randomisés ont évalué l'impact de l'ajout de kétamine à l'ACP par hydromorphone. Il y avait un large éventail de situations chirurgicales, notamment des chirurgies orthopédiques, thoraciques, cardiaques et abdominales. Globalement, cette méta-analyse a inclus des essais cliniques randomisés de bonne qualité avec une confiance modérée à haute dans les estimations.

L'ajout de kétamine a été associé à de faibles (de 4 à $13 \%$ ) diminutions des scores de douleur et réductions de la 
consommation de morphine (5 et $20 \mathrm{mg}$ au terme, respectivement, des premier et troisième jours postopératoires). Néanmoins, un effet analgésique dose-réponse de la kétamine n'a pas pu être établi avec certitude. Il n'y a pas eu d'augmentation des effets secondaires attendus de la kétamine (par exemple, hallucinations, dysphorie, diplopie, et stimulation sympathique). En outre, l'ajout de la kétamine a réduit l'incidence des nausées et vomissements postopératoires, bien qu'il y ait eu une mise en garde concernant des biais de publication concernant cette observation. Cela suggère que le nombre d'études publiées signalant une baisse des nausées et vomissements postopératoires avec l'utilisation de la kétamine a été supérieur au nombre attendu, par rapport aux études montrant l'absence ou l'augmentation d'effet de la kétamine sur l'incidence des nausées et vomissements postopératoires.

Des analyses systématiques antérieures ont décrit des constatations similaires concernant la baisse du besoin en opioïdes postopératoires avec l'utilisation de la kétamine. $^{9,10,12}$ Ces analyses antérieures font écho aux conclusions de Wang et coll. selon lesquelles les doses infra-anesthésiques de kétamine ont des effets secondaires minimes.

Cette analyse laisse quelques questions sans réponses et en soulève de nouvelles. Premièrement, quelle dose « infra-anesthésique » de kétamine devrait-on utiliser pour optimiser le bénéfice analgésique et minimiser les effets secondaires? Le terme «infra-anesthésique »implique simplement une dose insuffisante pour induire une anesthésie avec toutes ses composantes habituelles (c'est-à-dire l'amnésie, l'analgésie, la perte de connaissance et l'immobilité), mais aucun consensus sur une dose précise n'a été obtenu. Un schéma posologique fréquemment publié inclut un bolus lent de kétamine $\left(0,25\right.$ à $\left.0,5 \mathrm{mg} \cdot \mathrm{kg}^{-1}\right)$ avant l'incision, suivi d'une perfusion à un débit de $0,25-0,5 \mathrm{mg} \cdot \mathrm{kg}^{-1} \cdot \mathrm{h}^{-1}$ qui est interrompu une heure, environ, avant la fin de l'intervention chirurgicale. ${ }^{13}$ Il est important de reconnaître que cette dose est significativement inférieure à la limite supérieure retenue par Wang et coll. $\left(1,2 \mathrm{mg} \cdot \mathrm{kg}^{-1} \cdot \mathrm{h}^{-1}\right)$ dans leur méta-analyse pour décrire les doses infra-anesthésiques de kétamine. La sédation ou les autres effets psychotropes vus au cours de la période postopératoire sont rares avec cette plage de doses. Toutefois, nous manquons d'ECR évaluant la dose de kétamine en perfusion la plus adaptée pour l'efficacité et l'innocuité dans le contexte périopératoire.

Deuxièmement, pourquoi les essais cliniques randomisés et les analyses n'ont-ils pas établi une relation dose-réponse pour le bénéfice analgésique qu'apporte l'ajout de kétamine à l'ACP, en particulier chez les patients naïfs pour les opioïdes? Cela est-il dû à des limites méthodologiques (c'est-à-dire, essais cliniques randomisés avec des échantillons de petite taille, l'hétérogénéité dans les analyses systématiques et les méta-analyses) ou le rôle possiblement limité des récepteurs du NMDA dans la douleur postopératoire aiguë? Le rôle des récepteurs du NMDA dans le développement d'une sensibilisation centrale suggérerait certainement une prolifération/ régulation positive de ces récepteurs proportionnelle à la durée des douleurs ${ }^{14}$ et l'intérêt de la kétamine dans les états douloureux chroniques persistants tels que les douleurs neuropathiques, chroniques, d'origine cancéreuse et postopératoires. Ceci pourrait expliquer notre incapacité à exploiter le potentiel analgésique de la kétamine chez les patients en bonne santé au cours d'une période périopératoire relativement courte.

Troisièmement, les ERC inclus dans la méta-analyse de Wang et coll. ont-ils sous-déclaré les effets secondaires? La plupart des essais cliniques randomisés de leur étude étaient de taille modeste; La durée des études se limitait à la phase postopératoire précoce et, surtout, la majorité des ECR n'ont pas surveillé de manière rigoureuse la survenue d'effets secondaires de la kétamine. Cela dit, cette méta-analyse a montré une réduction de l'incidence des nausées et vomissements postopératoires chez les patients qui ont reçu la kétamine, une constatation également faite dans d'autres analyses. ${ }^{4,11}$ L'explication la plus plausible est une baisse de l'utilisation des opioïdes associée à la kétamine. Cette observation attire notre attention sur le problème important de l'évaluation de l'impact des ajouts analgésiques sur le soulagement des effets indésirables potentiels des opioïdes (par exemple : dépression respiratoire, hyperalgésie, tolérance, dépendance et rôle dans l'apparition des douleurs postopératoires persistantes). La baisse de l'intensité et de la fréquence de ces effets indésirables devrait être évaluée en tant que critères d'évaluation principaux dans des essais cliniques dotés d'une puissance suffisante.

Quatrièmement, quelles sont les implications possibles à long terme, positives (par exemple : réduction des douleurs postopératoires persistantes) et négatives (par exemple : trouble cognitif avec utilisation prolongée ou abus à long terme) de l'utilisation de la kétamine dans la période périopératoire? ${ }^{2,15}$ Bien qu'on ait suggéré que la kétamine était une arme importante dans l'arsenal thérapeutique de l'analgésie préventive, ${ }^{7}$ ses avantages l'emportent-ils sur les risques?

Alors, quo vadimus? Où allons-nous? Peut-être des développements futurs devront-ils envisager l'utilisation de la kétamine chez des populations spécifiques «à risque » dans la période postopératoire. On peut citer comme exemples les patients recevant de fortes doses d'opioïdes qui sont susceptibles de présenter les caractéristiques d'une hyperalgie ou d'une tolérance induite par les opioïdes. ${ }^{16}$ Les récepteurs du N-méthyl-D-aspartate sont impliqués dans la pathogénie de ces phénomènes. D'autres personnes 
pouvant bénéficier de l'administration de kétamine sont celles qui présentent des caractéristiques de douleurs neuropathiques ${ }^{17}$ (par exemple, les patients subissant une intervention sur la colonne vertébrale pour douleur axiale et/ou radiculaire) $)^{18,19}$ ou celles qui ont des antécédents d'effets secondaires significatifs (par exemple, des nausées et vomissements postopératoires) dus aux morphiniques. Nous devons également envisager d'utiliser la kétamine chez des personnes susceptibles de développer des douleurs postopératoires persistantes d'origine neuropathique. On peut citer comme populations à risque pour ce syndrome les patients subissant une mastectomie, une amputation et une thoracotomie. La kétamine peut être utile comme adjuvant dans ce cadre, car elle peut réduire la douleur postopératoire aiguë (un facteur de risque important des douleurs postopératoires persistantes) par son action antagoniste sur le récepteur NMDA. ${ }^{20}$

L'antagonisme des récepteurs NMDA est également responsable des effets antidépresseurs de la kétamine, ${ }^{5}$ ouvrant une voie de recherche intéressante pour les patients atteints de dépression (infracliniques ou patente). Le traitement par kétamine entraînera-t-il une amélioration des symptômes dépressifs et des scores de douleurs lombaires? Les scores de douleurs seront-ils abaissés en raison des effets euphoriques connus de la kétamine? L'évaluation de l'humeur et de la dépression chez les patients subissant une chirurgie par l'utilisation d'outils validés et de techniques avancées d'imagerie cérébrale fonctionnelle, à la fois avant et après l'utilisation de kétamine, pourrait nous fournir des informations utiles sur le lien entre analgésie et état psychologique.

Ce serait également de la négligence de ne pas mentionner que le bénéfice mineur obtenu par l'ajout de kétamine doit être évalué à l'aune du risque d'effets secondaires (psychotropes et sympathomimétiques) au cours de la période postopératoire. Il existe aussi des données probantes de troubles des fonctions hépatiques et urinaires chez les personnes ayant abusé de kétamine ou ayant reçu de multiples perfusions. ${ }^{2}$

L'addition de quelques autres substances (magnésium, lidocaïne, gabapentinoïdes, agonistes alpha-2, notamment) ${ }^{21}$ à l'analgésie morphinique a été explorée récemment dans un cadre avec des résultats mitigés d'après les ECR et la recherche observationnelle évaluant ces additions. ${ }^{22}$ Les cliniciens souhaitant employer la variété d'ajouts aux opioïdes pour le traitement de la douleur aiguë postopératoire doivent se poser les questions suivantes. L'utilisation d'un ajout est-elle associée à un impact cliniquement pertinent sur la quantité et la qualité de la douleur? L'ajout d'un médicament supplémentaire entraîne-t-il une réduction des besoins pour d'autres analgésiques, en particulier de ceux qui sont associés à des effets secondaires significatifs dans les populations sensibles (par exemple, les patients pédiatriques, âgés ou instables sur le plan hémodynamique, ou encore ceux ayant une altération de la fonction hépatique ou rénale)? Le médicament ajouté a-t-il un profil favorable en termes d'effets secondaires? Autrement dit, les effets secondaires fréquents du médicament ont-ils un impact minime et les effets indésirables graves sont-ils rares? L'administration du médicament ajouté est-elle faisable? Le médicament ajouté peut-il être administré en bolus unique ou en perfusion? Les deux méthodes sont-elles nécessaires? Y a-t-il de possibles interactions pharmacocinétiques ou pharmacodynamiques avec d'autres médicaments (par exemple : acétaminophène, anti-inflammatoires non stérö̈diens, morphiniques, antibiotiques)? Le médicament ajouté peut-il être utilisé pour une analgésie préventive? ${ }^{7}$ Les « co-domaines » présents de la douleur sont-ils favorablement affectés par le médicament ajouté? On a montré que l'anxiété, la dépression et le catastrophisme étaient de nature à pronostiquer, exacerber et perpétuer les douleurs postopératoires sévères. $^{23}$ L'utilisation d'outils validés $^{2}$ pour mesurer ces traits de personnalité avant et après l'intervention aiderait à comprendre les les bases « mécaniques » de l'action analgésique d'un médicament.

Bien que la kétamine et d'autres adjuvants aient la capacité d'améliorer l'analgésie périopératoire, nous continuons à chercher un adjuvant efficace idéal qui apporterait un bénéfice analgésique maximum et des effets indésirables minimums. Nous avons également besoin d'un équilibre entre les questions spécifiques à la procédure et les questions spécifiques au patient lorsque nous planifions des schémas thérapeutiques analgésiques périopératoires. Cela nécessite des essais cliniques bien conçus disposant d'une puissance suffisante, qui impliquent l'utilisation d'outils validés pour mesurer tous les aspects importants de la douleur périopératoire.

Conflicts of interest None declared.

Conflits d'intérêts Aucun déclaré.

\section{References}

1. Petrenko AB, Yamakura T, Baba H, Shimoji $K$. The role of $\mathrm{N}$-methyl-D-aspartate (NMDA) receptors in pain: a review. Anesth Analg 2003; 97: 1108-16.

2. Niesters $M$, Martini C, Dahan A. Ketamine for chronic pain: risks and benefits. Br J Clin Pharmacol 2014; 77: 357-67.

3. Hirota K, Lambert DG. Ketamine: new uses for an old drug? Br J Anaesth 2011; 107: 123-6.

4. Bell RF, Eccleston C, Kalso E. Ketamine as adjuvant to opioids for cancer pain. A qualitative systematic review. J Pain Symptom Manage 2003; 26: 867-75.

5. Iadarola ND, Niciu MJ, Richards EM, et al. Ketamine and other $\mathrm{N}$-methyl-D-aspartate receptor antagonists in the treatment of 
depression: a perspective review. Ther Adv Chronic Dis 2015; 6: 97-114.

6. Wang L, Johnston B, Kaushal A, Cheng D, Zhu F, Martin J. Ketamine added to morphine or hydromorphone patientcontrolled analgesia for acute postoperative pain in adults: a systematic review and meta-analysis of randomized trials. Can $\mathbf{J}$ Anesth 2016; 63: this issue: DOI: 10.1007/s12630-015-0551-4.

7. McCartney CJ, Sinha A, Katz J. A qualitative systematic review of the role of $\mathrm{N}$-methyl-D-aspartate receptor antagonists in preventive analgesia. Anesth Analg 2004; 98: 1385-400.

8. Subramaniam K, Subramaniam B, Steinbrook RA. Ketamine as adjuvant analgesic to opioids: a quantitative and qualitative systematic review. Anesth Analg 2004; 99: 482-95.

9. Elia N, Tramer MR. Ketamine and postoperative pain-a quantitative systematic review of randomised trials. Pain 2005; 113: 61-70.

10. Carstensen M, Moller AM. Adding ketamine to morphine for intravenous patient-controlled analgesia for acute postoperative pain: a qualitative review of randomized trials. Br J Anaesth 2010; 104: 401-6.

11. Laskowski K, Stirling A, McKay WP, Lim HJ. A systematic review of intravenous ketamine for postoperative analgesia. Can $\mathrm{J}$ Anesth 2011; 58: 911-23.

12. Bell RF, Dahl JB, Moore RA, Kalso E. Perioperative ketamine for acute postoperative pain. Cochrane Database Syst Rev 2006; 1 : CD004603.

13. Himmelseher $S$, Durieux $M E$. Ketamine for perioperative pain management. Anesthesiology 2005; 102: 211-20.

14. Woolf CJ, Thompson SW. The induction and maintenance of central sensitization is dependent on N-methyl-D-aspartic acid receptor activation; implications for the treatment of post-injury pain hypersensitivity states. Pain 1991; 44: 293-9.
15. Morgan CJ, Dodds CM, Furby H, et al. Long-term heavy ketamine use is associated with spatial memory impairment and altered hippocampal activation. Front Psychiatry 2014; 5: 149.

16. Loftus $R W$, Yeager MP, Clark JA, et al. Intraoperative ketamine reduces perioperative opiate consumption in opiate-dependent patients with chronic back pain undergoing back surgery. Anesthesiology 2010; 113: 639-46.

17. Dworkin RH, O'Connor AB, Kent $J$, et al; International Association for the Study of Pain Neuropathic Pain Special Interest Group. Interventional management of neuropathic pain: NeuPSIG recommendations. Pain 2013; 154: 2249-61.

18. Urban MK, Ya Deau JT, Wukovits B, Lipnitsky JY. Ketamine as an adjunct to postoperative pain management in opioid tolerant patients after spinal fusions: a prospective randomized trial. HSS J 2008; 4: 62-5.

19. Barreveld AM, Correll DJ, Liu X, et al. Ketamine decreases postoperative pain scores in patients taking opioids for chronic pain: results of a prospective, randomized, double-blind study. Pain Med 2013; 14: 925-34.

20. Dahl JB, Kehlet H. Preventive analgesia. Curr Opin Anaesthesiol 2011; 24: 331-8.

21. Schug SA, Goddard C. Recent advances in the pharmacological management of acute and chronic pain. Ann Palliat Med 2014; 3: 263-75.

22. Lui $F, N g K F$. Adjuvant analgesics in acute pain. Expert Opin Pharmacother 2011; 12: 363-85.

23. Brummett CM, Clauw DJ. Flipping the paradigm: from surgeryspecific to patient-driven perioperative analgesic algorithms. Anesthesiology 2015; 122: 731-3. 